Triterpenos e Outros Constituintes dos Frutos de Enterolobium contortisiliquum (Vell.) Morong (Fabaceae)

\author{
Miranda, M. L. D.; ${ }^{*}$ Garcez, F. R.; Garcez, W. S.
}

Rev. Virtual Quim., 2015, 7 (6), 2597-2605. Data de publicação na Web: 21 de outubro de 2015

http://www.uff.br/rvq

\title{
Triterpenes and Other Constituents from Fruits of Enterolobium contortisiliquum (Vell.) Morong (Fabaceae)
}

Abstract: From the fruits of specimen of Enterolobium contortisiliquum Fabaceae, ten known compounds were isolated, namely seven triterpenes, maslinic acid (1), betulinic acid (2), 3-oxo- $\beta$-amyrin (3), ursolic acid (4), $\beta$-amyrin (5), lupeol (6) and squalene (7); one steroid, ergosterol peroxide (8); one caffeic acid derivative, ethyl caffeate (9) and the ficaprenol-12 (10). This is the first reported occurrence of triterpenes 1-4 and 7, steroid 8 and ficaprenol-12 in the genus Enterolobium. The structures of the isolated compounds were determined on the basis of their spectral data.

Keywords: Enterolobium contortisiliquum; toxic plant; triterpenes.

\section{Resumo}

A partir dos frutos de um espécime de Enterolobium contortisiliquum Fabaceae, foram isolados dez compostos conhecidos, sendo sete triterpenos, o ácido maslínico (1), o ácido betulínico (2), 3-oxo- $\beta$-amirina (3), ácido ursólico (4), $\beta$-amirina (5), lupeol (6) e o esqualeno (7); um esteroide, peróxido de ergosterol (8); um derivado do ácido cafeico, cafeato de etila (9) e o ficaprenol-12 (10). Este é o primeiro relato dos triterpenos 1-4 e 7, do esteroide 8 e do ficaprenol-12 no gênero Enterolobium. As estruturas dos compostos isolados foram determinadas com base nos seus dados espectrais.

Palavras-chave: Enterolobium contortisiliquum; planta tóxica; triterpenos.

* Instituto Federal de Educação, Ciência e Tecnologia Goiano, Rede Arco Norte, Polo de Inovação em Agrocombustíveis, CP 66, CEP 75901-970, Rio Verde-GO, Brasil.

$M$ mayker.miranda@ifgoiano.edu.br

DOI: 10.5935/1984-6835.20150154 


\title{
Triterpenos e Outros Constituintes dos Frutos de Enterolobium contortisiliquum (Vell.) Morong (Fabaceae)
}

\author{
Mayker L. D. Miranda, ${ }^{a, *}$ Fernanda R. Garcez, ${ }^{b}$ Walmir S. Garcez ${ }^{b}$ \\ a Instituto Federal de Educação, Ciência e Tecnologia Goiano, Rede Arco Norte, Polo de \\ Inovação em Agrocombustíveis, CP 66, CEP 75901-970, Rio Verde-GO, Brasil.

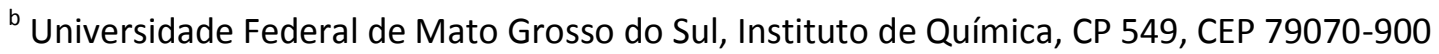 \\ Campo Grande-MS, Brasil. \\ * mayker.miranda@ifgoiano.edu.br
}

Recebido em 5 de agosto de 2015. Aceito para publicação em 21 de outubro de 2015

\section{Introdução}

\section{Parte Experimental}

2.1. Instrumentação e procedimentos

2.2. Material vegetal

2.3. Extração e isolamento dos constituintes químicos

\section{Resultados e Discussão}

\section{Conclusão}

\section{Introdução}

O gênero Enterolobium compreende diversas espécies nativas no Brasil, incluindo E. contortisiliquum, E. timbouva, E. gummiferum e E. shombungkii. ${ }^{1}$

Enterolobium contortisiliquum (Vell.) Morong, uma árvore de até $20-35 \mathrm{~m}$ de altura, é conhecida popularmente como ximbuva, orelha de negro, orelha de macaco, timburí, tamboril, timbaíba, timbó, pacará e tamburé, sendo encontrada em diversas regiões, desde Pará até o Rio Grande do Sul ${ }^{2}$ e seus frutos são conhecidos pela toxidez em gado. $^{3}$

Estudos fitoquímicos com a espécie
Enterolobium contortisiliquum revelaram a ocorrência de metabólitos secundários, como monoterpenos, identificados no óleo essencial de sementes, ${ }^{4}$ destacando-se principalmente saponinas já determinadas ${ }_{6}$ como triterpênicas, presentes no pericarpo.

Dando continuidade à nossa linha de pesquisa que visa conhecer a composição química de plantas tóxicas para o gado ocorrentes em Mato Grosso do Sul, o presente trabalho aborda $\mathrm{o}$ estudo fitoquímico de frutos de um espécime de $E$. contortisiliquum (Figura 1), que levou ao isolamento de dez compostos, destacando-se sete triterpenos, um esteroide, um derivado do ácido cafeico e um poliprenol. 


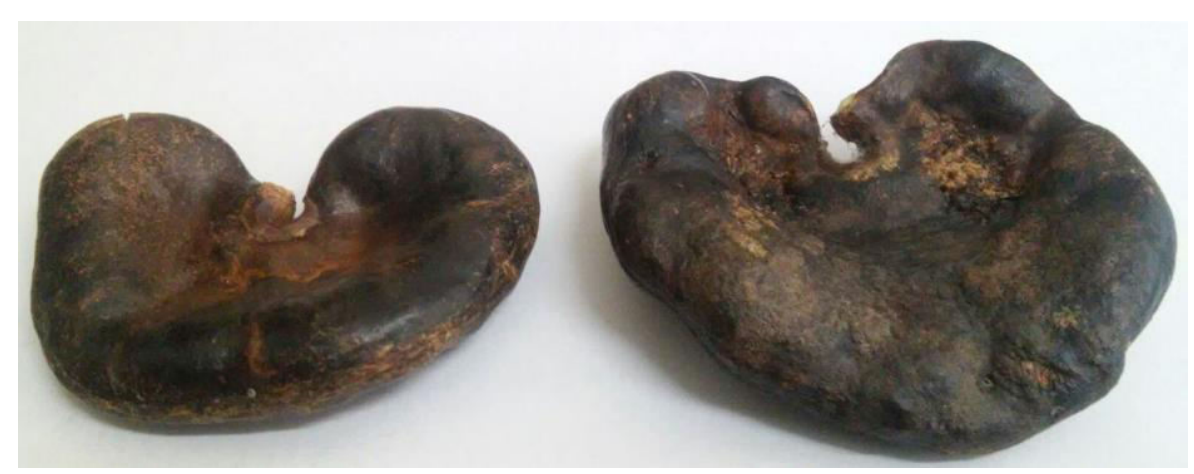

Figura 1. Frutos de Enterolobium contortisiliquum (Fabaceae)

\section{Parte Experimental}

\subsection{Instrumentação e procedimentos gerais}

Para as técnicas de cromatografia em coluna foram utilizadas como fases estacionárias gel de sílica 70-230 mesh e 230400 mesh. A quantidade de fase estacionária, o diâmetro e a altura do leito estão explicitadas no item 2.3. As análises por cromatografia em camada fina foram realizadas utilizando-se gel de sílica $60 \mathrm{GF}_{254} \mathrm{e}$ cromatofolhas de alumínio $60 \mathrm{~F}_{254}$ da Merck. Como reveladores foram utilizados sulfato de cério $\left[\mathrm{Ce}\left(\mathrm{SO}_{4}\right)_{2}\right]$ - solução a $2 \%$ em $\mathrm{H}_{2} \mathrm{SO}_{4}$ e luz $U_{254}$. Os espectros de RMN foram obtidos em espectrômetro Bruker ${ }^{\circ}$ DPX-300 $(300 / 75 \mathrm{MHz})$ e como solventes foram utilizados $\mathrm{CDCl}_{3}$ e $\mathrm{CD}_{3} \mathrm{OD}$.

\subsection{Material vegetal}

Os frutos de E. contortisiliquum foram coletados em janeiro de 2012, no município de Aquidauana, Mato Grosso do Sul, Brasil. A identificação botânica da espécie foi efetuada pelo Dr. A. R. Pott (DBI/UFMS), sendo uma exsicata incorporada ao Herbário CGMS, da UFMS, sob n ${ }^{\circ} 4021$.
1.3. Extração e isolamento dos constituintes químicos

Os frutos $(2 \mathrm{Kg})$ foram triturados $\mathrm{e}$ submetidos à extração com etanol, à temperatura ambiente, por uma semana. A solução resultante após filtração foi concentrada sob pressão reduzida até consistência xaroposa, fornecendo o extrato etanólico bruto $(400 \mathrm{~g})$. Este foi submetido a partições líquido-líquido entre metanol/água (9:1) e hexano, seguido por acetato de etila, dando origem a três resíduos após evaporação dos solventes: hexânico (30 g), acetato de etila ( $35 \mathrm{~g}$ ) e hidrometanólico ( 80 g).

O material obtido na fase hexânica foi submetido à cromatografia em coluna de gel de sílica 70-230 mesh (50,0 g; 10,0 X 60,0 $\mathrm{cm})$, eluída com misturas de Hex:AcOEt em gradiente de polaridade crescente (10:0, 9:1, 8,2, 7:3, 5:5, 0:10), originando 14 frações (ECFH-1 a ECFH-14) de $200 \mathrm{~mL}$ cada. Parte da fração ECFH-2 (180 mg) foi submetida à cromatografia em coluna de gel de sílica 200400 mesh (15,0 g; 3,0 X 40,0 cm), eluída com Hex:AcOEt em gradiente de polaridade crescente (95:5 a 80:20 e depois 0:100), originando 80 frações de $10 \mathrm{~mL}$ cada. Este processo resultou no isolamento das substâncias 6 (11,0 mg; 8:2) e 7 (25,0 mg; 95:5). A fração ECFH-3 (150 mg) foi submetida à cromatografia em coluna de gel de sílica 200-400 mesh (15,0 g; 3,0 X 40,0 $\mathrm{cm})$, eluída com Hex:AcOEt em gradiente de 
polaridade crescente (95:5 a 70:30 e depois $0: 100$ ), originando 100 frações de $10 \mathrm{~mL}$ cada. Este processo resultou no isolamento da substância 8 (10,0 mg; 8:2). A fração ECFH-4 (150 mg) foi submetida à cromatografia em coluna de gel de sílica 200400 mesh (15,0 g; 3,0 X 40,0 cm), eluída com Hex:AcOEt em gradiente de polaridade crescente (95:5 a 70:30 e depois 0:100), originando 120 frações de $10 \mathrm{~mL}$ cada. Este processo resultou no isolamento das substâncias 2 (8,0 mg; 9:1) , 3 (5,0 mg; 8:2) e 5 (6,7 mg; 8:2). O estudo da fração ECFH-5 $(100,0 \mathrm{mg})$ através do mesmo procedimento realizado para fração ECFH-4, levou ao isolamento do composto 10 (14,0 mg; 98:2). A fração ECFH-7 (100,0 mg) foi fracionada também em gel de sílica 230-400 mesh (15,0 g; 3,0 X 40,0 cm), eluída com Hex:AcOEt em gradiente crescente de polaridade, levando ao isolamento do composto 1 (5,0 mg; 40:60) e 4 (7,0 mg; 8:2). A fração ECFH-10 (160,0 $\mathrm{mg}$ ) foi submetida à cromatografia em coluna de gel de sílica 200-400 mesh (15,0 g; 3,0 X $40,0 \mathrm{~cm})$ utilizando como solvente AcOEt:MeOH em ordem crescente de polaridade, levando ao isolamento do composto composto 9 (12,0 mg; 0:100).

\section{Resultados e Discussão}

$\mathrm{O}$ espectro de RMN de ${ }^{1} \mathrm{H}$ da substância 1 revelou sete singletos em $\delta_{H} 0,69 ; 0,72 ; 0,82$; 0,$84 ; 0,89 ; 0,93$ e 1,05 indicando a presença de sete grupos metílicos. Apresentou também um singleto largo em $\delta_{H} \quad 5,19$ atribuído a hidrogênio olefínico, um dubleto em $\delta_{\mathrm{H}} 2,88(J=9,5 \mathrm{~Hz})$ e um duplo dubleto em $\delta_{H} 3,29(J=9,5$ e $4,5 \mathrm{~Hz})$ atribuído a hidrogênios oximetínicos.
A análise criteriosa do espectro de RMN ${ }^{13} \mathrm{C}$ revelou a presença de trinta átomos de carbono, dos quais sete são referentes a carbonos metílicos, nove metilênicos, seis metínicos e oito carbonos não hidrogenados. Estes dados indicaram que a substância 1 pertence à classe dos triterpenos pentacíclicos.

A presença do sinal em $\delta_{\mathrm{c}} 180,5$, atribuído a um carbono carboxílico, além dos sinais em $\delta_{\mathrm{c}} 122,0$ e $\delta_{\mathrm{c}} 143,8$ atribuídos a carbonos olefínicos, sendo um hidrogenado e o outro não hidrogenado, permitiram propor a presença do esqueleto oleanano com um grupo carboxila. A presença de sete grupos metílicos foi confirmada pelos sinais em $\delta_{\mathrm{C}}$ : 15,6 (C-24); 15,9 (C-25); 18,2 (C-26); 23,7 (C30); 26,9 (C-27); 29,4 (C-23); 34,6 (C-29).

Foram observados também sinais referentes a carbonos oximetínicos em $\delta_{\mathrm{c}}$ 68,2 e $\delta_{c} 83,2$ atribuídos aos carbonos C-2 e $\mathrm{C}-3$, respectivamente.

A configuração relativa dos grupos hidroxílicos em C-2 e C-3 foi atribuída com base nas constantes de acoplamento dos sinais dos hidrogênios oximetínicos. $O$ dubleto em $\delta_{\mathrm{H}} 2,88(J=9,5 \mathrm{~Hz})$ foi atribuído ao hidrogênio $\mathrm{H}-3$ em virtude de $\mathrm{H}-3$ acoplar somente com $\mathrm{H}-2$, visto que o carbono vizinho C-4 é não hidrogenado. A constante de acoplamento de $9,5 \mathrm{~Hz}$ permite dizer que H-3 está em posição axial. Da mesma forma, o duplo dubleto em $\delta_{\mathrm{H}} 3,29(J=9,5$ e $4,5 \mathrm{~Hz})$ foi atribuído a $\mathrm{H}-2$ por este estar acoplar-se a $\mathrm{H}-3$ e aos dois hidrogênios de C-1. A magnitude da constante de acoplamento permite dizer que $\mathrm{H}-2$ está em posição axial. Desta forma, pode-se afirmar que os grupos hidroxílicos de C-2 e C-3 estão em $\alpha$ e $\beta$, respectivamente (Figura 2 ). 


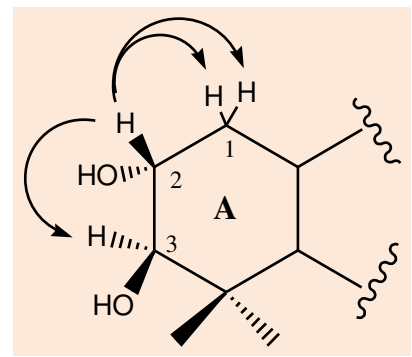

Figura 2. Acoplamentos ${ }^{1} \mathrm{H}^{-1} \mathrm{H}$ observados para o hidrogênio $\mathrm{H}-2$ pertencentes ao anel $A$ do composto 1

Com base no que foi discutido e em comparação com os dados já descritos na literatura, ${ }^{7}$ foi proposto para substância 1 a estrutura do ácido $2 \alpha, 3 \beta$-di-hidroxiolean-12ene-28-oico também conhecido como ácido maslínico (Figura 3).

No espectro de RMN de ${ }^{1} \mathrm{H}$ do composto 2 foram observados sinais característicos de seis grupos metílicos a $\delta_{\mathrm{H}} 0,76(s, \mathrm{H}-24) ; 0,82$ $(s, \mathrm{H}-25), 0,90(s, \mathrm{H}-27), 0,87(s, \mathrm{H}-23), 0,69$ ( $s$, $\mathrm{H}-26)$ e 1,63 (s, H-30). Um duplo dubleto atribuído a um átomo de hidrogênio ligado a carbono oxigenado a $\delta_{\mathrm{H}} 3,13(d d, J=5,4$ e $10,6 \mathrm{~Hz})$. Dois singletos largos a $\delta_{\mathrm{H}} 4,54(\mathrm{~s} / \mathrm{l}$ e $\delta_{H} 4,65(s /)$ referentes a um hidrogênio olefínico cada.

No espectro de RMN de ${ }^{13} \mathrm{C}$ foram listados trinta sinais que com o auxílio do DEPT 135 foram determinados como sendo: seis metilas, onze metilenos, seis metinos, seis carbonos não hidrogenados além de uma carboxila.

O cálculo do índice de deficiência de hidrogênio mostrou que a molécula apresenta seis insaturações, sendo cinco ciclos e uma ligação dupla evidenciada pelo RMN de ${ }^{13} \mathrm{C}$ através dos sinais a $\delta_{\mathrm{c}} 150,4$ (C) e $\delta_{C}$ 109,6 $\left(\mathrm{CH}_{2}\right)$. Um átomo de carbono oxigenado foi observado a $\delta_{c} 79,0$ e a carboxila em $\delta_{\mathrm{C}} 181,2(\mathrm{C}=0)$.

A união destes dados sugere que a substância 2 trata-se de um triterpeno ácido pentacíclico e pelos valores dos deslocamentos químicos de $\mathrm{RMN}{ }^{1} \mathrm{H}$ e ${ }^{13} \mathrm{C}$, confirma-se tratar do triterpeno ácido betulínico. ${ }^{8}$ (Figura 3 )
Os triterpenos 3-oxo- $\beta$-amirina (3), ácido ursólico (4), $\beta$-amirina (5), lupeol (6) e esqualeno (7) foram identificados, a partir das análises dos espectros de $\mathrm{RMN}{ }^{1} \mathrm{H}$ e ${ }^{13} \mathrm{C} \mathrm{e}$ comparação com os dados espectrais já existentes na literatura. ${ }^{9-13}$ As substâncias 8, 9 e $\mathbf{1 0}$ foram identificadas, a partir das análises dos espectros de $\mathrm{RMN}{ }^{1} \mathrm{H}$ e ${ }^{13} \mathrm{C}$ e comparação com amostras autênticas, como sendo o peróxido de ergosterol (8), ${ }^{14,15}$ cafeato de etila $(9)^{16}$ e ficaprenol-12 (10), ${ }^{17}$ (Figura 3). Os dados espectroscópicos dos compostos (1-7) isolados dos frutos de $E$. contortisiliquum podem ser conferidos na Tabela 1.

A seguir encontram-se os dados espectroscópicos dos compostos $\mathbf{8}$ a $\mathbf{1 0}$ :

Peróxido de ergosterol (8): óleo verde. RMN de ${ }^{13} \mathrm{C}\left(75 \mathrm{MHz}, \mathrm{CDCl}_{3}\right) \delta_{\mathrm{c}}: 30,1$ (C-1), 34,7 (C-2), 66,4 (C-3), 39,3 (C-4), 82,2 (C-5), 135,2 (C-6), 130,7 (C-7), 79,4 (C-8), 51,1 (C-9), 36,9 (C-10), 20,6 (C-11), 36,9 (C-12), 44,5 (C13), 51,6 (C-14), 23,4 (C-15), 28,6 (C-16), 56,2 (C-17), 12,8 (C-18), 18,2 (C-19), 39,7 (C-20), 20,8 (C-21), 135,4 (C-22), 132,3 (C-23), 42,7 (C-24), 33,1 (C-25), 19,6 (C-26), 19,9 (C-27), 17,5 (C-28).

Cafeato de etila (9): Cristal amarelo. RMN ${ }^{13} \mathrm{C}\left(75 \mathrm{MHz}, \mathrm{CDCl}_{3}\right) \delta_{\mathrm{C}}: 125,8$ (C-1), 114,3 (C2), 145,8 (C-3), 148,6 (C-4), 116,0 (C-5), 121,7 (C-6), 145,2 (C-7), 114,8 (C-8), 166,9 (C-9), 60,1 (C-1'), 14,4 (C-2').

Ficaprenol-12 (10): Óleo amarelo. $\mathrm{RMN}^{13} \mathrm{C}$ (75 MHz, CDCl $)$ ) $: 59,0$ (C-1), 124,9 (C-2), 139,9 (C-3), 32,0 (C-4), 31,9-32,2 (C-a), 26,326,7 (C-b), 124,1-124,4 (C-c), 131,2-136,1 (Cd), 39,7 (C-e), 25,7 (C-f), 17,7 (C-g), 16,0 (C-h), 23,4 (C-i), 23,7 (C-j). 
Tabela 1. Dados de RMN de ${ }^{13} \mathrm{C}\left(\mathrm{CDCl}_{3}, 75 \mathrm{MHz}\right)^{\mathrm{a}}$ e $\left(\mathrm{CD}_{3} \mathrm{OD}, 75 \mathrm{MHz}\right)^{\mathrm{b}}$ dos compostos $\mathbf{1}^{\mathrm{b}}, \mathbf{2}^{\mathrm{a}}$, $3^{\mathrm{a}}, 4^{\mathrm{a}}, 5^{\mathrm{a}}, 6^{\mathrm{a}}$ e $7^{\mathrm{a}}$

\begin{tabular}{|c|c|c|c|c|c|c|c|}
\hline & & & & $\delta_{c}$ & & & \\
\hline C & 1 & 2 & 3 & 4 & 5 & 6 & 7 \\
\hline 1 & 46,7 & 38,7 & 38,7 & 38,6 & 38,7 & 38,7 & 25,7 \\
\hline 2 & 68,1 & 27,4 & 27,2 & 27,9 & 27,2 & 27,4 & 131,2 \\
\hline 3 & 83,1 & 79,0 & 218,0 & 78,8 & 79,0 & 79,0 & 124,5 \\
\hline 4 & 39,4 & 38,8 & 38,5 & 38,6 & 38,5 & 38,8 & 26,7 \\
\hline 5 & 55,2 & 55,3 & 55,2 & 55,2 & 55,2 & 55,3 & 39,8 \\
\hline 6 & 16,5 & 18,3 & 18,4 & 18,2 & 18,4 & 18,3 & 134,9 \\
\hline 7 & 32,5 & 34,2 & 32,9 & 32,9 & 32,9 & 34,2 & 124,2 \\
\hline 8 & 41,5 & 40,7 & 38,7 & 39,3 & 38,7 & 40,6 & 26,7 \\
\hline 9 & 47,0 & 50,5 & 47,6 & 47,5 & 47,6 & 50,5 & 39,8 \\
\hline 10 & 37,9 & 37,0 & 37,2 & 36,8 & 37,2 & 37,0 & 135,1 \\
\hline 11 & 22,9 & 20,8 & 23,6 & 23,2 & 23,6 & 20,8 & 124,5 \\
\hline 12 & 122,0 & 25,5 & 121,7 & 125,5 & 121,7 & 25,5 & 28,3 \\
\hline 13 & 143,8 & 38,3 & 145,1 & 138,1 & 145,1 & 38,3 & 28,3 \\
\hline 14 & 41,2 & 42,4 & 41,7 & 41,9 & 41,7 & 42,4 & 124,5 \\
\hline 15 & 28,0 & 30,5 & 26,2 & 27,9 & 26,2 & 27,9 & 135,1 \\
\hline 16 & 23,0 & 32,2 & 27,2 & 24,1 & 27,2 & 37,2 & 39,8 \\
\hline 17 & 45,8 & 56,3 & 32,5 & 48,2 & 32,5 & 42,0 & 26,7 \\
\hline 18 & 41,2 & 46,8 & 47,2 & 52,7 & 47,2 & 49,2 & 124,2 \\
\hline 19 & 45,8 & 49,2 & 46,8 & 39,0 & 46,8 & 46,8 & 134,9 \\
\hline 20 & 30,3 & 150,4 & 31,1 & 38,8 & 31,1 & 150,9 & 39,8 \\
\hline 21 & 33,6 & 30,2 & 34,7 & 30,6 & 34,7 & 30,5 & 26,7 \\
\hline 22 & 32,5 & 37,1 & 36,9 & 36,8 & 36,9 & 39,0 & 124,5 \\
\hline 23 & 29,4 & 27,9 & 28,3 & 27,9 & 28,3 & 29,7 & 131,2 \\
\hline 24 & 15,6 & 15,3 & 15,5 & 15,3 & 15,5 & 15,4 & 25,7 \\
\hline 25 & 15,9 & 16,0 & 15,6 & 15,5 & 15,6 & 16,1 & 17,6 \\
\hline 26 & 18,2 & 16,1 & 16,8 & 16,9 & 16,8 & 15,9 & 15,9 \\
\hline 27 & 26,9 & 14,7 & 25,9 & 23,4 & 25,9 & 14,5 & 15,9 \\
\hline 28 & 180,5 & 181,2 & 28,4 & 180,6 & 28,4 & 18,0 & 16,0 \\
\hline 29 & 34,6 & 109,7 & 33,3 & 16,9 & 33,3 & 109,3 & 16,0 \\
\hline 30 & 23,7 & 19,3 & 23,7 & 21,0 & 23,7 & 19,3 & 17,6 \\
\hline
\end{tabular}

Ácido maslínico (1); Ácido betulínico (2); 3-oxo-6-amirina (3); Ácido ursólico (4); 6-amirina (5); Lupeol (6); Esqualeno (7). 

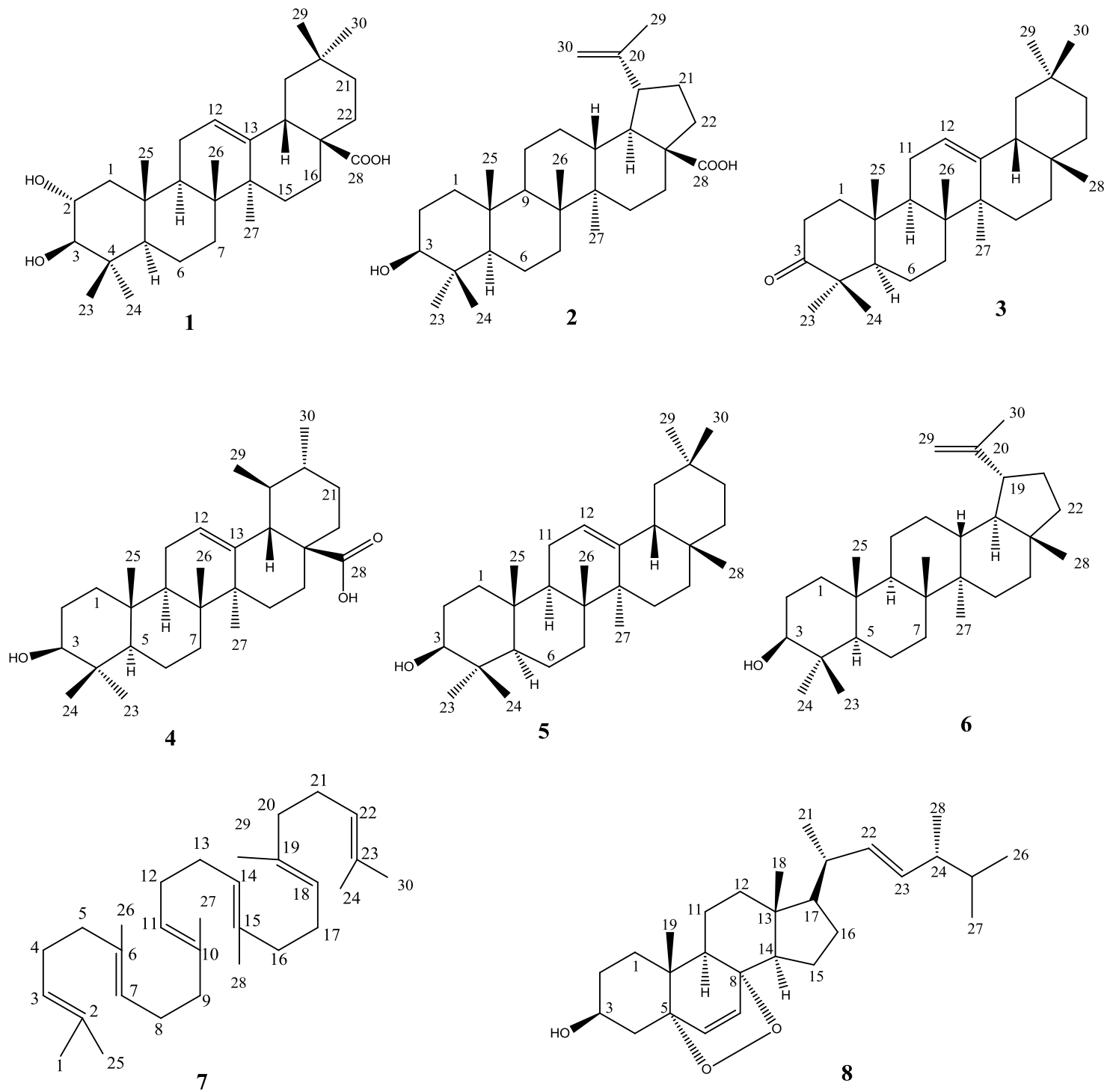<smiles>CCOC(=O)/C=C/c1ccc(O)c(O)c1</smiles>

9

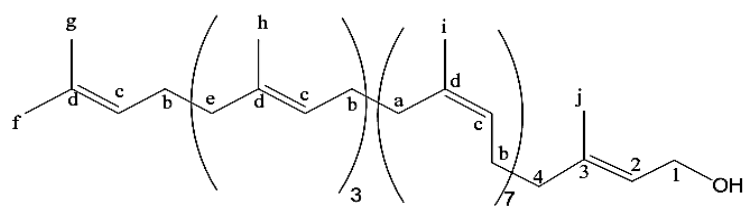

10

Figura 3. Estruturas dos constituintes químicos isolados do resíduo hexânico dos frutos de Enterolobium contortisiliquum (FABACEAE): ácido maslínico (1); ácido betulínico (2); 3-oxo- $\beta$ amirina (3); ácido ursólico (4); $\beta$-amirina (5); lupeol (6); esqualeno (7); peróxido de ergosterol (8); cafeato de etila (9) e ficaprenol-12 (10) 


\section{Conclusão}

O estudo fitoquímico do resíduo hexânico dos frutos de um espécime de Enterolobium contortisiliquum coletado no município de Aquidauana - MS levou ao isolamento de dez substâncias, destacando-se sete triterpenos: ácido maslínico (1), ácido betulínico (2), 3oxo- $\beta$-amirina (3), ácido ursólico (4), $\beta$ amirina (5), lupeol (6) e esqualeno (7). Exceto os triterpenos 5 e $\mathbf{6}$, os demais estão sendo relatados pela primeira vez no gênero Enterolobium. Os outros compostos foram: um esteroide, o peróxido de ergosterol (8); um derivado do ácido cafeico, o cafeato de etila (9) e um poliprenol, o ficaprenol-12 (10).

Considerando-se que as favas de $E$. contortisiliquum são relatadas como uma importante causa de intoxicação para bovinos causando impactos negativos na pecuária, ${ }^{18}$ os resultados obtidos no presente trabalho podem fornecer subsídios para estudos futuros visando à identificação do princípio ativo tóxico.

\section{Agradecimentos}

À FUNDECT-MS, CNPq, CAPES, CPqPROPP/UFMS e ao IF goiano.

\section{Referências Bibliográficas}

${ }^{1}$ Bonel-Raposo, J.; Riet-Correa, F.; Guim, T. N.; Schuch, I. D.; Grecco, F. B.; Fernandes, C. G. Intoxicação aguda e abortos em cobaias pelas favas de Enterolobium contortisiliquum (Leg. Mimosoideae). Pesquisa Veterinária Brasileira 2008, 28, 593. [CrossRef]

${ }^{2}$ Gadelha, I. C. N.; Câmara, A. C. L.; Silva, I. P.; Batista, J. S.; Melo, M. M.; Blanco, B. S. Toxic effect of the pericarp of the enterolobium contortisiliquum (Vell.) Morong fruits on chicks. International Journal of Applied Research in Veterinary 2015, 13, 135. [Link]
${ }^{3}$ Souza, R. I. C.; Santos, A. C.; Ribas, N. L. K. S.; Colodel, E. M.; Leal, P. V.; Pupin, R. C.; Carvalho, N. M.; Lemos, R. A. A. Doenças tóxicas de bovinos em Mato Grosso do Sul. Semina 2015, 36, 1355. [Link]

${ }^{4}$ Shahat, A. A.; El-barouty, G.; Hassan, R. A.; Hammouda, F. M.; Abdel-Rahman, F. H.; Saleh, M. A. Study of the anti-proliferative activity of 5-substituted 4,7-dimethoxy-1,3benzodioxole derivatives of SY-1 from Antrodia camphorate on human COLO 205 colon cancer cells. Journal of Environmental Science And Healt, Part B 2008, 43, 519. [Link]

${ }^{5}$ Mimaki, Y.; Harada, H.; Sakuma, C.; Haraguchi, M.; Yui, S.; Kudo, T.; Yamazaki, M.; Sashida, Y. Contortisiliosides A-G: Isolation of seven new triterpene bisdesmosides from Enterolobium contortisiliquum and cytotoxic activity. Helvetica Chimica Acta 2004, 87, 851. [CrossRef]

${ }^{6}$ Mimaki, Y.; Harada, H.; Sakuma, C.; Haraguchi, M.; Yui, S.; Kudo, T.; Yamazaki, M.; Sashida, Y. Enterolosaponins A and B, novel triterpene bisdesmosides from Enterolobium contortisiliquum, and evaluation for their macrophage-oriented cytotoxic activity. Bioorganic Medicinal Chemistry Letters 2003, 13, 623. [CrossRef] [PubMed]

${ }^{7}$ Lemes, G. F.; Ferri, P. H.; Lopes, M. N. Constituintes químicos de Hyptidendron canum (Pohl ex Benth.) R. Harley (Lamiaceae). Química Nova 2011, 34, 39. [CrossRef]

${ }^{8}$ Mahato, S. B.; Kundu, A. P. ${ }^{13}$ C NMR Spectra of pentacyclic triterpenoids a compilation and some saliente features. Phytochemistry 1994, 37, 1517. [CrossRef]

${ }^{9}$ Salama, A. M.; Avendaño, I. Y. Actividad antiinflamatoria de $\delta$-amirona y $4^{\prime}, 7$ dimetoxiapigenina de Alnus acuminata. Revista Colombiana de Ciencias Químico Farmacéuticas 2005, 34, 117. [Link]

${ }^{10}$ Junior, L. R. A.; Garcez, F. R.; Garcez, W. S.; Guterres, Z. R. Pregnanos e outros constituintes das raízes de Macrosiphonia petraea (A. St.-Hil.) Kuntze (Apocynaceae). Química Nova 2013, 36, 519. [CrossRef]

${ }^{11}$ Carvalho, M. G.; Velandia, J. R.; Oliveira, L. F.; Bezerra, F. B. Triterpenos isolados de 
Eschweilera longipes Miers (Lecythidaceae). Química Nova 1998, 21, 740. [CrossRef]

${ }^{12}$ Souza, A. D. L.; Rocha, A. F. I.; Pinheiro, M. L. B.; Andrade, C. H. S.; Galotta, A. L. A. Q.; Santos, M. P. S. S. Constituintes químicos de Gustavia Augusta L. (Lecythidaceae) Química Nova 2001, 4, 442. [CrossRef]

${ }^{13}$ Miranda, M. L. D.; Souza, A. F.; Rodrigues, E. D.; Garcez, F. R.; Garcez, W. S. Constituintes Químicos das Folhas de Riedeliella graciliflora Harms (Leguminosae). Química Nova 2012, 35, 1306. [CrossRef]

${ }^{14}$ Rocha, M. R.; Vieira, I. J. C.; Braz-Filho, R. Estudo Fitoquímico de Rauia nodosa (Rutaceae). Resumos da 37a Reunião Anual da Sociedade Brasileira de Química, Natal, Brasil, 2014. [Link]
${ }^{15}$ Yue, J.; Chen, S.; Lin, Z.; Sun, H. Sterols from the fungus Lactarium volemus. Phytochemistry 2001, 56, 801. [CrossRef]

${ }^{16}$ Gibbons, S.; Mathew, K. T.; Gray, A. I. A caffeic acid ester from Halocnemum strobilaceum. Phytochemistry 1999, 51, 465. [CrossRef]

${ }^{17}$ Aoki, T.; Matsuo, K.; Suga, T.; Ohta, S. Arachisprenols: Polyprenols possessing a geranyl residue from Arachis hypogaea. Phytochemistry 1997, 46, 715. [CrossRef]

${ }^{18}$ Costa, R. L. D.; Marine, A.; Tanaka, D.; Berndt, A.; Andrade, F. M. E. Um caso de intoxicação de bovinos por Enterolobium contortisiliquum (Timboril) no Brasil. Archivos de Zootecnia 2009, 58, 313. [CrossRef] 